\title{
PERFIL EPIDEMIOLÓGICO E MORBIMORTALDADE DOS PACIENTES SUBMETIDOS À RECONSTRUÇÃO DE TRÂNSITO INTESTINAL: EXPERIÊNCIA DE UM CENTRO SECUNDÁRIO DO NORDESTE BRASILEIRO
}

\author{
Epidemiologic profile and morbimortality of patients undergoing reconstruction intestinal \\ transit: experience of a secundary health service in the northeast of Brazil \\ Jeany Borges e SILVA, Djalma Ribeiro COSTA, Francisco Julimar Correia de MENEZES, \\ José Marconi TAVARES, Adryano Gonçalves MARQUES, Rodrigo Dornfeld ESCALANTE
}

Trabalho realizado no Serviço de Cirurgia Geral do Hospital Geral Dr. Waldemar de Alcântara, Fortaleza, CE, Brasil
DESCRTORES - Reconstrução. Complicações pós-operatórias. Indicadores de morbimortalidade.

\section{Correspondência:}

Jeany Borges e Silva,

e-mail jeanyborges@yahoo.com.br

Fonte de financiamento: não há

Conflito de interesses: não há

Recebido para publicação: 02/06/2010 Aceito para publicação: 01/07/2010

HEADINGS - Reconstruction. Postoperative complications. Indicators of morbidity and mortality.
RESUMO - Racional - A reconstrução do trânsito intestinal não está isenta de riscos cirúrgicos e apresenta taxas consideráveis de complicações pós-operatórias, sendo que a infecção continua a ser um dos maiores desafios existentes neste procedimento. Objetivo Perfil epidemiológico e morbimortalidade dos pacientes submetidos à reconstrução de trânsito intestinal. Métodos - Foram analisados retrospectivamente 86 prontuários de pacientes com colostomia ou ileostomia, através de fatores que tivessem impacto sobre a morbimortalidade após a reconstrução de trânsito intestinal, de janeiro de 2003 a abril de 2009. Resultados - Houve 20 mulheres e 60 homens, com idade média de 43 anos. A colostomia em alça $(n=34)$ e o trauma abdominal indicando colostomia ou ileostomia foram as condições mais frequentes. O intervalo médio entre a confecção do estoma e a reconstrução de trânsito intestinal foi 15,7 meses. O índice de morbidade foi $56,8 \%$, sendo a infecção incisional a complicação mais comum (27.47\%). A permanência hospitalar média foi 7,6 dias. Houve regressão linear positiva entre permanência hospitalar pós-operatória e a idade do paciente. Demonstrou-se associação estatisticamente significativa entre o prolongamento da permanência hospitalar e a ocorrência de complicações $(p<0,001)$. Conclusão - Pode-se inferir que ocorrência de complicações pós-operatórias e idade associaram-se a prolongamento da permanência hospitalar.

ABSTRACT - Background - The reconstruction of the intestinal tract is not surgical complications risk-free and is associated to postoperative complications high rates; furthermore, infection remains the hardest challenge in this procedure. Aim - Epidemiological profile and mortality and morbidity in patients undergoing reconstruction of intestinal transit. Methods - Retrospectively, 86 patients with intestinal stomas were analyzed through factors that impact on the morbimortality afterwards intestinal transit reconstruction, since January 2003 to April 2009. Results - Loop colostomy ( $n=34)$ and abdominal trauma implicating $38.2 \%$ of indications to colostomy or ileostomy, were the most frequent conditions. The mean interval between stoma confection and intestinal transit reconstruction was 15.7 months. The morbidity frequency was $56.8 \%$ and incisional infection was its commonest complication (27.47\%). The mean inpatient length of stay was 7.6 days. There was positive linear regression between post-operative inpatient length of stay and inpatient's age. Inpatient length of stay prolongation is associated to occurrence of complications $(p<0,001)$. Conclusion -It can be inferred that the occurrence of postoperative complications and age were associated with prolonged hospital stay.

\section{INTRODUÇÃO}

$\mathrm{D}$ esde a primeira descrição da realização de colostomia pelo francês Littré ${ }^{11}$, a utilização de estomas e suas indicações foram modificadas conforme a evolução da medicina. Sua utilização como auxílio terapêutico das afecções colorretais é bem definido, porém a morbimortalidade associada à reconstrução do trânsito intestinal é ainda motivo de preocupações ${ }^{1}$.

A confecção de um estoma é procedimento comum, realizado 
por diversas especialidades cirúrgicas, sobretudo em situações de urgência, visando à redução da morbimortalidade pós-operatória ${ }^{10}$.

A reconstrução do trânsito intestinal não está isenta de riscos cirúrgicos e apresenta taxas consideráveis de complicações pós-operatórias, sendo que a infecção continua a ser um dos maiores desafios existentes neste procedimento ${ }^{4}$. Relatam-se na literatura taxas de morbidade variando de 0 a $50 \%$ e de mortalidade 0 a $4,5 \%{ }^{1}$.

Assim, este artigo tem como objetivo analisar as características demográficas, a mortalidade e morbidade associada ao procedimento de reconstrução de trânsito intestinal em um ambiente de hospital secundário, relacionar a permanência hospitalar com as características epidemiológicas do paciente e com o número de complicações pós-operatórias.

\section{MÉTODOS}

Estudo retrospectivo de uma série de 86 pacientes portadores de ostomias intestinais (cólon e intestino delgado) encaminhados pela rede de atendimento público do estado de Ceará, no período compreendido entre janeiro de 2003 a abril de 2009.

Analisaram-se as seguintes variáveis: sexo e idade dos pacientes; a indicação e os tempos de permanência hospitalar e da ostomia; complicações pós-operatórias e óbitos.

Realizaram-se estatísticas descritiva e inferencial através dos testes Qui-quadrado de Pearson e regressão linear.

Os dados foram agrupados no programa Microsoft Office Excel 2007TM e todas as análises realizadas pelo Medcalc v8.0TM. O nível de significância considerado foi $p<0,05$.

\section{RESULTADOS}

A idade média foi de 43 anos, e houve predomínio do sexo masculino (76,74\%) (Tabela 1). Lesões traumáticas (38,2\%) e câncer colorretal (22,47\%) foram as indicações de ostomização mais frequentes (Tabela 2). Perfuração por arma de fogo, com 58,82\% dos casos, foi a principal lesão traumática motivadora de ostomização. A colostomia em alça foi o tipo de ostomia preferencialmente realizada $(38,46 \%$ dos pacientes) (Tabela 2). O tempo médio de permanência com a ostomia, antes do seu fechamento, foi de 15,7 meses com variação de três a 284 meses ( $\chi 2: 278,355$ $\mathrm{DF}=3, \mathrm{p}<0,0001$ ) (Tabela 1).

O índice de morbidade global foi de $56,80 \%$ $(n=52)$, sendo a infecção incisional a condição mórbida mais documentada, acometendo $27,47 \%$ dos pacientes (Tabela 3).

Foram realizadas seis relaparotomias (6,59\%), sendo três por evisceração, uma por deiscência de
TABELA 1 - Características clínico-demográficas dos 86 pacientes ostomizados

\begin{tabular}{|c|c|c|c|c|c|c|c|}
\hline \multicolumn{2}{|l|}{ Características } & Média $\pm D P$ & Mediana & Intervalo & $\mathrm{N}$ & $\%$ & $p$ \\
\hline Sexo & Homem & & & & 66 & 77 & \\
\hline & Mulher & & & & 20 & 23 & $P=0,02$ \\
\hline Idade & & $\begin{array}{c}43 \pm 18,2 \\
\text { anos }\end{array}$ & & $\begin{array}{l}12-83 \\
\text { anos }\end{array}$ & 86 & 100 & \\
\hline $\begin{array}{l}\text { Tempo de } \\
\text { ostomia }\end{array}$ & & $\begin{array}{c}15,56 \pm 44,99 \\
\text { meses }\end{array}$ & 9 meses & $\begin{array}{l}\text { 3-384 } \\
\text { meses }\end{array}$ & 71 & 77 & $P<0,0001$ \\
\hline $\begin{array}{l}\text { Tempo de } \\
\text { permanência } \\
\text { hospitalar }\end{array}$ & & $\begin{array}{c}7,57 \pm 6,42 \\
\text { dias }\end{array}$ & 5 meses & $2-45$ dias & 86 & 94,5 & $P<0,0001$ \\
\hline
\end{tabular}

DP: desvio-padrão. N: número de casos. Teste do Qui-quadrado para distribuição da normalidade

TABELA 2 - Causas para indicação da ostomia

\begin{tabular}{|l|c|c|}
\hline & $N^{\circ}$ de casos & $\%$ \\
\hline Trauma & 20 & \\
\hline Perfuração por arma de fogo & 5 & \\
\hline Perfuração por arma branca & 2 & \\
\hline Trauma abdominal fechado & 2 & \\
\hline Trauma perineal & & 22,47 \\
\hline Câncer colorretal & & $16,85 \%$ \\
\hline Abdome agudo inflamatório & 4 & \\
\hline Perfuração intestinal & 3 & \\
\hline Diverticulite & 1 & \\
\hline Apendicite & & 5,62 \\
\hline Abdome agudo obstrutivo & 4 & \\
\hline Volvo de Sigmóide & 1 & \\
\hline Hérnia encarcerada & & $4,94 \%$ \\
\hline Reintervençães & 3 & \\
\hline Deiscência de anastomose & 1 & \\
\hline Estenose e deiscência da anastomose & & $12,35 \%$ \\
\hline Outras & 2 & \\
\hline Síndrome de Fournier & 2 & \\
\hline Iatrogenia (pós-curetagem uterina) & 1 & \\
\hline Iatrogenia (pós-anexectomia) & 2 & \\
\hline Proteção de anastomose & 1 & \\
\hline Hérnia interna & 1 & \\
\hline Tumor gástrico com necrose de cólon & 1 & \\
\hline Doença de Hirschsprung &
\end{tabular}

TABELA 3 - Tipos de ostomias realizadas

\begin{tabular}{l|c|c} 
& $\mathrm{N}^{\circ}$ de casos & $\%$ \\
\hline Colostomia em alça & 33 & 36,26 \\
\hline Hartmann & 27 & 29,67 \\
\hline Ileostomia em alça & 15 & 16,4 \\
\hline Ileostomia terminal & 7 & 7,69 \\
\hline Mikulicz & 2 & 2,19 \\
\hline Cecostomia & 2 & 2,19 \\
\hline Colostomia em alça + fístula mucosa & 2 & 2,19 \\
\hline Ileostomia em alça + hartmann & 1 & 1,10 \\
\hline Ileostomia em alça + fistula mucosa & 1 & 1,10 \\
\hline Hartmann + fístula mucosa & 1 & 1,10 \\
\hline
\end{tabular}

ferida operatória, uma por hematoma de ferida e uma por estenose da anastomose, sendo submetido à nova ileostomia. A taxa de insucesso nas reconstruções de trânsito foi de 6,59\%. A perda de seguimento após alta hospitalar foi de $9,89 \%$. Sete pacientes $(7,69 \%)$ evoluíram em pós-operatório tardio com hérnia incisional. Não houve registro de óbito (Tabela 4). 
TABELA 4 - Morbidade global entre 86 pacientes

\begin{tabular}{l|c|c|} 
& $\mathbf{N}^{\circ}$ de complicações & $\%$ \\
\hline Infecção incisional & 25 & $27,40 \%$ \\
\hline Deiscência de ferida & 11 & $12,08 \%$ \\
\hline Fístula êntero-cutânea & 5 & $5,49 \%$ \\
\hline Evisceração & 3 & $3,30 \%$ \\
\hline Abscesso intra-cavitário & 2 & $3,30 \%$ \\
\hline Deiscência de anastomose & 3 & $2,20 \%$ \\
\hline Fístula retovaginal & 1 & $1,01 \%$ \\
\hline Hematoma de ferida & 1 & $1,01 \%$ \\
\hline Estenose de anastomose & 1 & $1,01 \%$ \\
\hline Total & $\mathbf{5 2}$ & $\mathbf{5 6 , 8 0 \%}$ \\
\hline
\end{tabular}

Os pacientes tiveram tempo médio de permanência hospitalar no pós-operatório de 7,6 dias, variando de dois a 45 dias $(P<0,0001,(\chi 2: 86,037 D F=6)$. (Tabela 1$)$. A permanência hospitalar no pós-operatório apresentou curva de regressão linear positiva em função da idade $(P=0,001)$ (Figura 1).

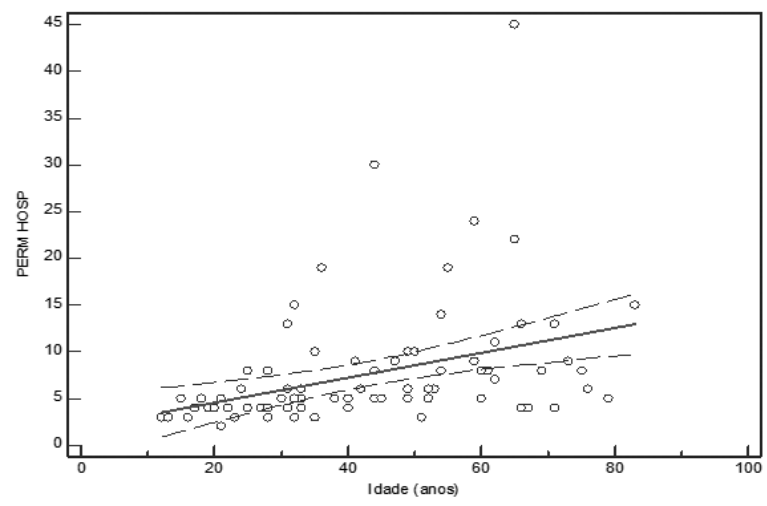

FIGURA 1 - Regressão linear da permanência hospitalar após reconstrução de trânsito intestinal em função da idade

Utilizando-se do teste do Qui-quadrado, houve relação significativa entre a permanência hospitalar e o número de complicações $(P<0,001, \chi 2: 19,31 \mathrm{DF}=1)$ (Tabela 5).

TABELA 5 - Comparação entre o número de pacientes com complicações após reconstrução de trânsito e a permanência hospitalar

\begin{tabular}{lcccc}
\hline & \multicolumn{4}{c}{$\begin{array}{c}\text { Complicações pós- } \\
\text { operatórias na RTI }\end{array}$} \\
\hline & & Sim & Não & Total \\
\hline Tempo de & $>7,6$ dias & 22 & 6 & 28 \\
permanência & $<7,6$ dias & 15 & 43 & 58 \\
hospitalar & Total & 37 & 49 & 86 \\
\hline
\end{tabular}

\section{DISCUSSÃO}

Em um estudo retrospectivo de 46 pacientes submetidos a fechamento de colostomia houve média de 41,8 anos, semelhante a encontrada no presente estudo, mas com predominância do sexo feminino, o que diverge desta casuística9 .
A reconstrução do trânsito intestinal está associada a índices elevados de morbimortalidade. Bocic et al. ${ }^{5}$ estudaram 132 pacientes portadores de ileostomias e colostomias que foram submetidos à reconstrução do trânsito intestinal, relatando incidência de morbidade da ordem de $36,2 \%$ e mortalidade de $1,7 \%$. Bannura et al. ${ }^{2}$, estudando 100 pacientes submetidos à reconstrução do trânsito intestinal, observaram índice de morbidade de $34 \%$ e mortalidade de $1,7 \%$. HabrGama et al. ${ }^{8}$, avaliando 73 pacientes submetidos à reconstrução do trânsito intestinal após colostomia à Hartmann observaram índice de complicações de $34,2 \%$, sendo a infecção da ferida operatória a mais frequente $(12,2 \%)$, e as fístulas com $8,2 \%$; a mortalidade observada nesta série foi de 3,6\%. Carreiro et al. ${ }^{6}$, estudando 35 pacientes portadores de colostomias em alça, após lesões traumáticas de reto e que foram submetidos ao seu fechamento, observaram taxa global de complicações de $25,7 \%$, sendo a infecção da ferida operatória a mais frequente com 17,1\%; não houve óbitos nesta série. Curi et al. ${ }^{7}$, avaliando 67 pacientes portadores de estomas intestinais observaram índice de morbidade de $19,2 \%$, sendo o abscesso de parede $(10,4 \%)$ a complicação mais comum. Na presente casuística, observou-se a taxa global de complicações de $56,8 \%$ após o procedimento de reconstrução do trânsito intestinal, níveis esses que poderiam ser justificados pela alta morbidade associada à ostomia pós-trauma. Em um estudo retrospectivo de 40 pacientes submetidos à colostomia pós-trauma, houve taxa significativa de morbidade de $30 \%$ dos pacientes ${ }^{3}$.

O trauma é a causa mais comum de indicação das ostomias ${ }^{7}$, e o presente artigo corrobora com esta informação; o trauma abdominal foi a principal causa. Houve predomínio das colostomias em alças (36,26\%) em detrimento das ileostomias (24,09\%), apesar de haver tendência, cada vez maior, em optar-se pela ileostomia ao invés das clássicas colostomias ${ }^{6}$.

O tempo médio entre a confecção e o fechamento das ostomias foi de 15,7 meses, superior ao encontrado na literatura consultada de 6,9 meses $^{9}$. A presente casuística revelou regressão linear positiva estatisticamente significativa da permanência hospitalar no pós-operatório de reconstrução de trânsito intestinal em função da idade, bem como relação também estatisticamente significativa do número de complicações com a permanência hospitalar no pósoperatório de reconstrução intestinal.

\section{CONCLUSÃO}

O procedimento de reconstrução intestinal está associado à alta morbidade, sendo que a ocorrência de complicações pós-operatórias e a idade dos pacientes estão diretamente relacionadas com o prolongamento do tempo de permanência hospitalar. 


\section{REFERÊNCIAS}

1. Bahten LCV, Nicoluzzi JEL, Silveira F, Nicollelli, GM, Kumagai LY, De Lima VZ. Morbimortalidade da Reconstrução de Transito Intestinal Colônica em Hospital Universitário - Análise de 42 Casos. 2006; 26(2): 123-127.

2. Bannura GC, Perales CG, Contreras JP, Valencia $C E$, Melo CL. Reconstituición de tránsito intestinal luego de la operación de Hartmann: análisis de 100 pacientes. Rev Chil Cir 1999; 51(4): 359-66.

3. Berne JD, Velmahos GC, Chan LS, Asensio JA, Demetriades D. The high morbidity of colostomy closure after trauma: further support for the primary repair of colon injuries.

4. Biondo-Simões MLP, Brenner S, Lemos R, Duck D, Rey SD. Análise das complicações pós-operatórias em decolostomias. Acta Cir Bras 2000; 15(3): 53-57.

5. Bocic GA, Jensen CB, Abedrapo MM, Garrido RC, Pérez GO, Cúeneo AZ. Colostomías e ileostomias: 8 anos de experiência clínica. Rev Hosp Clin Univ Chile 1999; 10(3): 195-200.

6. Carreiro PRL, Lázaro da Silva A, Abrantes WL. Fechamento precoce das colostomias em pacientes com trauma do reto: Um estudo prospectivo e casualizado. Rev. Coleg. Bras. Cir 2000; 27 (5): 298-304.
7. Curi A, Moreira Júnior $H$, Mascarenhas JCS, Moreira JPT, Almeida AC, Azevedo IF, Louza LR, Moreira H. Morbimortalidade associada à reconstrução de trânsito intestinal. - Análise de 67 casos. Rev Bras Coloproct 2002; 22(2): 88-97.

8. Habr-Gama A, Teixeira MG, Vieira MJF, Miléu LF, Laurino Neto R, Pinotti HW. Operação de Hartmann e suas conseqüências. Rev Bras Colo-proctologia 1997; 17(1): 5-10.

9. Khoury DA, Beck DE, Opelka FG, Hicks TC, Timmcke AE, Gathright JB Jr. Colostomy closure. Ochsner clinic experience. Dis Colon Rectum. 1996 Jun; 39(6): 605-9.

10. Santos CHM; Bezerra MM; Bezerra FMM; Paraguassú BR. Perfil do paciente ostomizado e complicações relacionadas ao estoma. Rev bras. colo-proctol. 2007. 27(1): 16-19.

11.Stumm EMF, Oliveira ERA, Kirschner RM. Perfil de pacientes ostomizados, Scientia Medica. 2008; 18(1)26-30. 